\title{
Shape Matters: Modelling, Calibrating \& Validating Pedestrian Movement Considering Groups
}

\author{
Luca Crociani $^{a 1}$, Yiping Zeng ${ }^{b}$, Giuseppe Vizzari $^{a}$ and Stefania Bandini ${ }^{a, c}$ \\ ${ }^{a}$ Complex Systems and Artificial Intelligence research center \\ Department of Informatics, Systems and Communication, University of Milano-Bicocca, \\ Milan, Italy \\ ${ }^{b}$ State Key Laboratory of Fire Science, University of Science and Technology of China, \\ Hefei, Peoples Republic of China \\ ${ }^{c}$ Research Center for Advanced Science and Technology, The University of Tokyo, \\ Tokyo, Japan
}

\begin{abstract}
Computer simulation for pedestrian dynamics is at the same time an application area in which research has completed its cycle and an active and lively research context in which contributions from different disciplines still produce advancements on the state of the art. The study of effects of the presence of groups in the simulated population is object of growing interest in the community. While previous results have started to investigate the phenomenon, implying conflicting tendencies for pedestrians, mechanisms for the flexible management of cohesion among group members are still investigated both in continuous and discrete modelling efforts. The present effort is aimed at extending previous modelling efforts, preserving and improving the capability to generate overall plausible aggregated dynamics, while at the same time improving the precision in the microscopic group dynamics, with particular attention to the shape of dyads. The paper presents validated results based on empirical data from a controlled experiment, also discussing qualitative results in different common situations (bends and bottlenecks).
\end{abstract}

Keywords: pedestrian simulation, social groups, multi-agent systems 2010 MSC: $68 \mathrm{U} 20$

\footnotetext{
${ }^{1}$ Corresponding author; luca.crociani@disco.unimib.it

Preprint submitted to Simulation Modelling Practice and Theory

May 15, 2018
} 


\section{Introduction}

Computer simulation systems for the study of pedestrian dynamics is at the same time an application area in which research has completed its cycle, producing technological transfer, and an active and lively area in which contributions from different disciplines still produce advancements on the state of the art. While computer simulations imply a significant simplification and reduction of the overall complexity of the phenomena taking place in crowded environments, they actually produce useful information about plausible usage of the environment in normal conditions that planners, designers and crowd managers actually use in everyday activities [1].

A point that has been recently attracting relevant attention both as a factor able to influence both the low level, basic locomotion aspect of pedestrians, as well as higher level decisions related to the planning of paths within the environment, is represented by the presence of groups in the simulated population. Despite having been at least touched by observations in the early 2000s (see, e.g., [2]), simulation models facing this further element adding up to the complexity of the analyzed phenomenon started to be defined and studied several years later.

Whereas previous results have started to investigate the phenomenon, implying conflicting tendencies for pedestrians (i.e. walk fast and efficiently, but stay close to some pedestrians [3]), mechanisms for the flexible management of cohesion among group members are still investigated both in continuous and discrete modelling efforts. The possibility to preserve the overall shape of the group, in terms of walking patterns [3, that flexibly shift according to external conditions (mainly pedestrian density, presence of obstacles), preserving overall good aggregate properties of the models (e.g. generating plausible fundamental diagrams) is still an open issue. In a previous modelling effort [4] we described an adaptive mechanism for the regulation of the movement of group members in order to preserve group cohesion. While this contribution was able to produce quantitatively reasonable results, in particular with reference to the overall level 
of dispersion of a group in low to medium density conditions [5], this model is not really considering the actual shape of the group in the course the movement in the environment. The present effort is aimed at extending the previous model, preserving and improving the capability to generate overall plausible aggregated dynamics, while at the same time improving the precision in the microscopic group dynamics, with particular attention to the shape of dyads. This aspect, in fact, is calibrated and validated using empirical data from a controlled experiment performed in 2015 in a corridor setting [6]. The presented model is therefore providing overall plausible results, that are validated according to low-medium density conditions, that can generate plausible hypotheses about the implications of groups in higher density conditions, to be tested in real world experiments and/or observations. The overall aim of the work is to propose an approach, a model, and a specific set of values for the relevant parameters for which this model is directly applicable within a relatively large set of scenarios (i.e. low to medium pedestrian density, irrespectively of the geometry of the environment) producing plausible results. We will, consequently, show results covering a wide range of situations, in particular levels of density, accepting a relatively contained error in specific situations, also due to the discrete nature of the adopted approach. The long term observation and results described in [7, for instance, clearly show that (at least within that specific cultural context, that is, a Japanese multipurpose building including a commercial area - for which the authors later provided an even richer and more detailed analysis in [8]) interpersonal distances among members of dyads are not always compatible with a discrete modelling effort: across the observed densities, in most situations dyad members are walking at a distance between 47 and $65 \mathrm{~cm}$, whereas in a discrete modelling approach this value could be either 40 or $80 \mathrm{~cm}$ (in case of a discretization in $40 \mathrm{~cm}$ sided cells). On the other hand, the discrete approach grants us interesting computational properties, in particular a linear growth of simulation costs with respect to the number of simulated pedestrians, and (empirically) the possibility to manage an interesting number of simulated pedestrians within real-time constraints. 
The paper breaks down as follows: we first introduce a more systematic discussion of the relevant literature, then Section 3 formally introduces the proposed model, including a discussion of its limitations. The process and experimentation for calibration, validation and analysis of achieved results is then given. Conclusions and future works end the paper.

\section{Related Works}

The number and variety of different researches and practical applications focused or involving pedestrian and crowd simulation makes it really hard to provide a compact but comprehensive overview of the area. Only considering the academic and scientific context, we must consider that there are completely dedicated scientific events (such as the International Conference on Pedestrian and Evacuation Dynamics, that reached its eight edition in 2016). However, to try to organize different contributions in the field, a first broad characterization of modelling efforts lies in the choice of the analytical unit: macroscopic models (see, e.g. 9]) do not really consider the single pedestrian, but they rather compute the evolution of aggregated scalar or vectorial measurements, such as velocity and density, in the different points of the simulated environment; microscopic models, on the other hand, compute the dynamic changes in the position of the individual pedestrians in time 2 Among microscopic models three main categories can be defined according to how pedestrians are considered: the first category represents pedestrians as particles subject to forces (whose most successful representative is the social force model [11]), the second as states of cells in which the environment is subdivided (for Cellular Automata (CA) approaches, whose most general representative is represented by the floor field model [12]), or autonomous agents acting and interacting in an environment that can be either continuous or discrete (agent-based approaches). Agent based approaches rep-

\footnotetext{
${ }^{2}$ There is no consensus on an exact definition of mesoscopic models, although they generally consider individual pedestrians but often provide a coarse grained representation of the environment [10].
} 
resent the most heterogeneous category: works like [13] and [14 that essentially extend CA approaches, separating the pedestrians from the environment and granting them a behavioural specification that is generally more complex than what is generally represented in terms of a simple CA transition rule, but they essentially adopt similar methodologies; other approaches like [15, 16] are more aimed at generating visually effective and believable pedestrians and crowds in virtual worlds; finally, works like [17, employ cognitive agent models for different goals (not only related to movement), but they are not generally aimed at making predictions about pedestrian movement for sake of decision support.

All approaches implicitly adopt fundamental concepts from proxemic [18], like the tendency of a pedestrian to stay away from other ones while moving towards his/her goal, although few authors actually mention this anthropological theory (notably CA based models [19, 20] and an agent-based model [21]). However, proxemics also mentions the fact that, besides keeping strangers at a distance, pedestrians also allow acquanintances, friends, family members to reach a shorter distance. Basically, a dynamic interpretation of the proxemic principles accounts for the effect of the presence of groups of pedestrians, whose members will, at the same time, try to stay close to other members while staying at a distance from other pedestrians.

A relatively small number of recent works represent a relevant effort towards the modelling of groups, respectively in particle-based [22, 23, 24], in CA-based [25], and in agent-based approaches [26, 27, 28]. All the above mentioned approaches interpret the impact of groups by means of additional contributions to the overall pedestrian behaviour representing the tendency to stay close to other group members. Moreover, they mostly deal with small groups in relatively low density conditions; those dealing with relatively large groups (tens of pedestrians) were not validated against real data.

A second wave of works related to groups was mainly aimed at gathering in a more systematic way evidences on the implications of the presence of groups within a population of moving pedestrians. [7] describes a simple particle based model based on the notion of potential representing the discomfort that the 
pedestrian feels when moving to a certain position: the model is calibrated and validated against a large scale dataset proving information about the movement of pedestrians and groups within a shopping center. 29] performed an experimental observation of the implications of the presence of groups in a higher density evacuation from a room. [30] performed a similar kind of experiment, but also considering the implication of group membership on the decision of which exit to use, in a situation in which multiple exits are available. The model presented in 4 provides an adaptive mechanism for the regulation of the movement of group members in order to preserve group cohesion, but the model is not really considering the actual shape of the group in the course the movement in the environment. All these works agree on the fact that group members move slower than individuals, and that the difference in velocity is weakly affected by density (although no study provides a systematic analysis of the behaviour of groups in high density situations); only some works (most notably [7]) actually study the dynamic changes in the shape of the group with varying density. Nonetheless, this aspect, and particularly the flexibility of group members in temporarily accepting a different spatial arrangement, might have a serious effect on the overall smoothness of pedestrian flows in case of locally high densities, due to the presence of obstacles or particular environmental constraints (e.g. bottlenecks or bends). Although a recent contribution describing the (social) pshycological stance on the current simulation models considering groups considers them relatively limited 31] (and this is understandable, considering the disciplinary perspective), current results show that this line of work can lead both to an increased understanding of the overall phenomena related to pedestrian dynamics as well as potential technological transfers to systems currently employed by designers, planners, and crowd managers.

\section{The simulation model}

The simulation model here presented is an extension of the model already described in [4] and [32, performed to grant a more valid and reliable simulation 
of pedestrian and group dynamics. Previous works by the authors, in fact, have highlighted the quality of the behaviour of pedestrian groups by comparing simulations with quantitative data on their dispersion and speed. The present work will extend the previous results by focusing specifically on the preservation the shape of groups of two members, to better reflect empirical observations, and by providing a more systematic and thorough calibration and validation process. As it will be discussed in next section, the experimental procedures will be accurately simulated and calibration weights of the model will be selected to fit the observations performed by the authors in 2015 [6] with a minimal error.

The section breaks down as the following: firstly, the model components previously introduced with other works by the authors (see [4] and 32 for a thorough discussion) will be briefly described; the discussion of the novel components and mechanisms will follow, emphasizing their motivations and improvement with respect to the baseline.

\subsection{Environmental structure}

The adopted agent environment [33] is discrete and modelled with a grid of $40 \mathrm{~cm}$ sided square cells. Each cell is denoted as $c$ while we refer to its coordinates, as for the coordinates of an agent, in a vectorial fashion $\vec{x}_{c}$. The size is chosen to consider the average area occupied by a pedestrian [34, and also respecting the maximum densities usually observed in real scenarios. The cells have a state that informs the agents about their movement possibilities: each one can be vacant or occupied by obstacles or at most two pedestrians, so as to be able to manage locally high density situations [35].

To allow the configuration of a pedestrian simulation scenario, several markers are defined with different purposes. This set of objects has been introduced to allow the movement at the operational level and the reasoning at the tactical level, identifying intermediate and final targets for agents' plans:

- start areas, generating agents in the simulation;

- obstacles; 
- openings, sets of cells that divide the environment into regions together with obstacles. These objects are fundamental elements supporting agents' decisions, representing intermediate destinations for route choice activities;

- regions, objects that are not being considered by agents during the simulation, but they instead describe properties of the region where they are located. Through them it is possible to design particular elements of the environment (e.g. stairs, waiting areas) implying special behaviours;

- final destinations of agents, generally implying their removal from the simulation.

The classic floor fields approach [36] supports agents' navigation, using the environment as a container of information used for the interactions between entities. In this particular model, discrete potentials are spread from cells of obstacles and destinations, informing about distances to these objects. The two types of floor fields are denoted as path field, spread from openings and final destinations (one per destination object), and obstacle field, a unique field spread from all the cells marked as obstacle. In addition, a dynamic floor field that has been denoted as proxemic field is used to reproduce a proxemic behaviour [37] in a repulsive sense, allowing agents to preserve acceptable distances from other agents. This approach generates a plausible navigation of the environment as well as an anthropologically founded means of regulating interpersonal distances among pedestrians.

\subsection{Movement of agents: basic locomotion}

A pedestrian agent $a$ is defined as a n-uple $a=\left\langle G, \nu_{a}^{*}, \vec{x}_{a}, \vec{\nu}_{a}\right.$, CurrentDest, FinalDest $\rangle$. $G$ is the set of agents composing the possible group of the pedestrian $(G=\emptyset$ if the pedestrian does not belong to a group). Other parameters respectively define: the desired speed $\nu_{a}^{*}$ of the agent, the current position $\vec{x}_{a}$ and current velocity $\vec{\nu}_{a}$, the intermediate and final destination identifiers, used to select the relative path field. The population of agents in the simulation is 


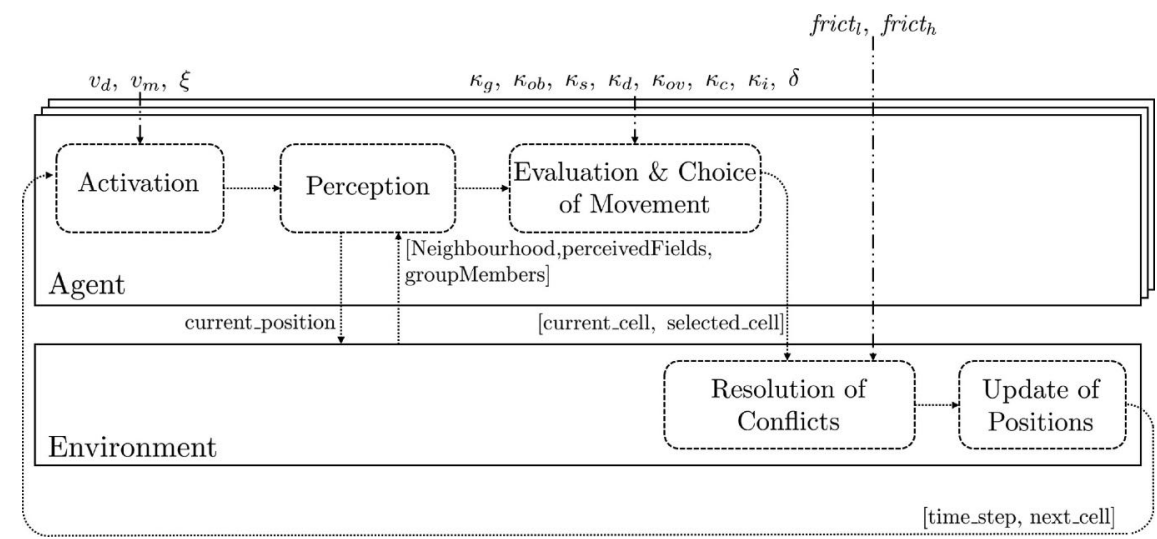

Figure 1: Tasks executed at each step of the simulation by agents and the environment. Parameters of the model are grouped for each task and shown as input at the top of the figure. Possible multiple outputs of a task are represented inside square brackets.

referred with the set $A$. The workflow for the agent to reproduce its movement at each step is illustrated in Figure 1.

The first activity that the agent performs is the Activation for the locomotion at the current step. While this task seems completely simple and elementary, it is determinant for the model since it contains the mechanisms developed for the reproduction of heterogeneous speeds and acceleration of agents from a zero speed.

If the locomotion is activated at the current step, the choice of the next movement is performed with an utility function, evaluating position of the current target, nearby obstacles and pedestrians, other pedestrians of the group and previous direction of movement. The utility value associated to each cell of the neighbourhood of the agent determines its probability to be chosen by the agent, according to the function $P(c)=N \cdot e^{U(c)}$. The update of the position is then executed after the conflict resolution phase.

\subsubsection{Activation and reproduction of speed}

The reproduction of different speeds is allowed in a probabilistic way, with a perspective that implies each agent to have a probability $\psi$ to be activated 


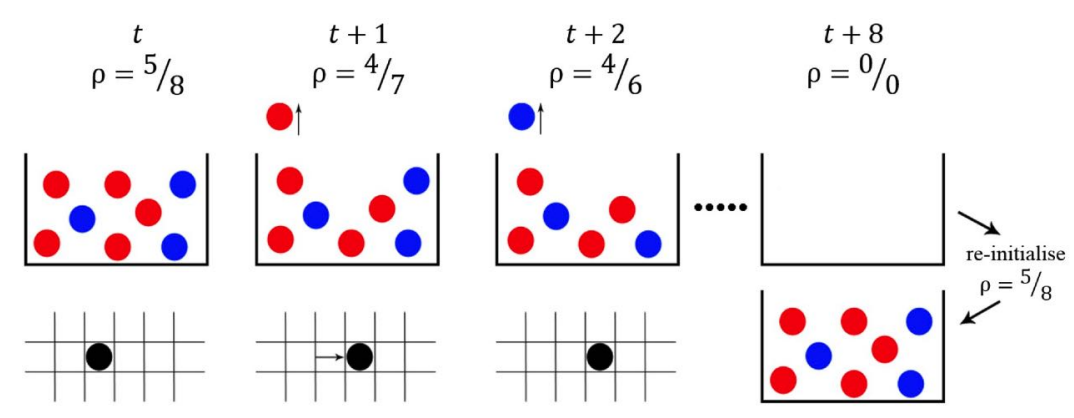

Figure 2: Example of the functioning of the probabilistic method to manage speeds. The agent is configured with $\nu_{a}^{*}=1 \mathrm{~m} / \mathrm{s}$, while $\nu_{\max }=1.6 \mathrm{~m} / \mathrm{s}$.

for the movement at each time step of the simulation. This means that fastest agents will have $\psi=1$ leading them to move at the maximum speed for the whole population $\nu_{\max }=\frac{\Lambda}{\mathcal{T}} \mathrm{m} / \mathrm{s}$, where $\Lambda$ is the length of the stride performed in one time step and $\mathcal{T}$ is the assumed duration of the simulation turn.

In this simulation model, the agents are moving in discrete position of a rectangular grid and using the Moore neighbourhood of 1 cell. The distance $\Lambda$ defining the speed $\nu_{\max }$, thus, differs among linear $\Lambda_{l}=0.4 \mathrm{~m}$ and diagonal $\Lambda_{d}=0.4 \cdot \sqrt{2}$ movement. As discussed and empirically investigated in [38, the mechanisms defining the stochastic reproduction of speeds of this model are able to overcome the issue and approximate $\nu_{\max }$ to $\frac{\Lambda_{l}}{\mathcal{T}}$.

Overall, the paradigm used in this model differs from other applied methods in this field, which generally employ a coarser time discretization and make pedestrians to perform more than one stride over one turn of the simulation (see, e.g., [39, 40]). The introduction of the probability $\psi$, indeed, allows the reproduction of the desired speed $\nu_{a}^{*}<=\nu_{\max }$ of an agent $a$. In particular, $\psi_{a}$ is calculated as:

$$
\psi_{a}=\frac{\nu_{a}^{*}}{\nu_{\max }}
$$

While $\nu_{\max }$ is calculated with $\Lambda_{l}$ and $\mathcal{T}$ which are model parameters, the 
desired speed of the agent can be also differentiated with values $\in \mathbb{R}$. The management of $\nu_{a}^{*}$ with a probabilistic mechanism can make the simulated speed of the agent in a fixed and relatively small time window quite different from the configured one, due to the randomness. To improve this, the probability $\psi$ is managed dynamically as an extraction-without-replacement mechanism, which limits the duration of the time window needed to synchronize the simulated speed with $\nu_{a}^{*}$. The algorithm is exemplified in Figure 2 The configuration of $\nu_{a}^{*}=1 \mathrm{~m} / \mathrm{s}$ and $\nu_{\max }=1.6 \mathrm{~m} / \mathrm{s}$ provides $\psi=\frac{5}{8}$. This generates an initial set of 5 activate and 3 do-not-activate events. Every 8 simulation turn, the simulated speed of the agent is equal to $1 \mathrm{~m} / \mathrm{s}$ without diagonal movements. In case of diagonal movements, the simulated speed of the agent is higher than expected and thus the set of events is enlarged with a number $\Delta_{\text {diag }} \in \mathbb{R}$ of do-not-activate events calculated as:

$$
\Delta_{\text {diag }}=\frac{\nu_{\max }}{\nu_{a}^{*}} \cdot(\sqrt{2}-1)
$$

$\Delta_{\text {diag }}$ will describe the additional number of time steps for which the agent has to yield its position in order to synchronize again with its $\nu_{a}^{*}$.

In 38 it is shown how the algorithm is able to reproduce arbitrary velocities $\in \mathbb{R}$. A feature that is not explained in Fig. 2 (for sake of space and simplicity) is that the set of activate/do-not-activate events can be also updated depending on what happens during the simulated dynamic. In particular if the agent has extracted an activate event, but at the end of the step it was not able to move then the event is put back in the set. In parallel, when the agent moves in a diagonal neighbour covering a distance equal to $\sqrt{2} \cdot 0.4 \mathrm{~m}$, the set is enlarged with a partial do-not-move event whose size is proportional to the desired speed of the agent (see 38, for full details about this aspect.

The paradigm illustrated above is suitable for the consideration of another microscopic effect of the human motion that, though, has not been considered until this point. When a persons stops his/her motion, due to encountering obstacles or congestion, he/she generally needs some time before reaching again 
the desired walking speed. This is due to pure mechanical elements related to the locomotion, on one hand, but also to what can be denoted as reaction time, describing the interval of time passing from the occurrence of an event (e.g. some space just got free after being occupied by another person) and the beginning of the action execution.

The delay just introduced is abstracted with the parameter $\xi$, which represents a novel component of this model. In particular, $\xi$ describes the amount of time an agent will need to wait before being activated (and therefore move) right after halting due to conflicts or impossibility of movement in case of congestion. The effects that this additional mechanism produces on the overall dynamics are in a sense similar to related techniques proposed in the literature (e.g. [39, 41]) since all of them generally make certain positions of the environment less probably usable (or completely not usable) for a certain amount of time. More precisely, the proposed approach will act and affect the distance between an agent and the one directly in front, assuming the case of 1-directional movement. On the other hand, this element is here modelled with a different perspective, identifying the reasons of this delay related to the capabilities of the pedestrian. The effects of this mechanism will be more clearly discussed in Section 4.2 but the expectation is that the overall flow should be mostly affected in low to medium densities, since in low densities pedestrians are mostly freely moving, with few stops triggering the $\xi$ and in high densities pedestrians are frequently unable to move due to congestion, and not immediately exploiting a movement opportunity actually relieves extremely severe congestions.

While the following components of the model define the way the agent chooses its next movement, according to possible group members and the configuration of the environment, the parameter $\xi$ is crucial for the reproduction of the physics of the system. This will be analysed in Section 4 where the validation of this part of the model is presented based on fundamental diagram and flow analysis in bottleneck scenarios. 


\subsubsection{Evaluation and choice of movements}

The evaluation of the possible movements is performed by means of the utility function $U(c)$ of a destination cell $c$, which takes the form of a weighted sum of components associated to the reproduction of a particular behaviour:

$$
U(c)=\frac{\kappa_{g} G(c)+\kappa_{o b} O b(c)+\kappa_{s} S(c)+\kappa_{c} C(c)+\kappa_{i} I(c)+\kappa_{d} D(c)+\kappa_{o v} O v(c)}{d}
$$

where $d$ is the distance of the new cell from the current position, that is 1 for cells in the Von Neumann neighbourhood and $\sqrt{2}$ for diagonal cells. The first three functions combine information derived by local floor fields and they model the basic factors considered in the pedestrian behaviour: goal attraction (i), geometric (ii) and social repulsion (iii). The fourth and fifth elements aggregate the perceived positions of members of pedestrian group, both simple (iv) and structured (v), to calculate the level of attractiveness of each neighbour cell, relating to cohesion phenomenon. The idea of structured groups has been firstly introduced to model large groups which split among subgroups of the simple type, but as it will be shown in Sec. 3.3 , a structured group is also formed for the simulation of counter-flows, in order to stimulate the lane formation.

The final two elements of the sum represent preferences with respect to movement, helping the model to reproduce more realistic simulations both in qualitative and quantitative perspective: (vi) adds a bonus to the utility of the cell next to the pedestrian according to his/her previous direction, while (vii) describes the overlapping mechanism, a method used to allow our model the possibility to treat high density situations, allowing two pedestrians temporarily occupying the same cell at the same step. Moreover, the overlapping is allowed only in case of counter-flow situations.

Calibration is allowed with $\kappa_{g}, \kappa_{o b}, \kappa_{s}, \kappa_{d}, \kappa_{o v}, \kappa_{c}, \kappa_{i} \in \mathbb{R}$. After the utility evaluation for all the cells of the neighbourhood, the choice of action is stochastic, with the probability to move in each cell $c$ as ( $N$ is the normalization factor):

$$
P(c)=N \cdot e^{U(c)}
$$




\subsubsection{Resolution of conflicts and execution of the movement}

The choice of movement is parallel for the whole set of agents, thus conflicts related to the choice of the same destination cell must be solved with further rules, deciding whether the agent can finalize its movement or not. In this model we use the concept of friction as possible outcome of the conflict. This concept has been introduced in [42, 43] and also analyzed in [44, and it implies that all pedestrians involved in the conflict keep their position at the end of the time-step. The rule is then refined to include also the possibility that the conflict is solved with the movement of two pedestrians in the destination cell, only in case of agents in a counter-flow condition.

\subsection{Modelling groups of pedestrians}

To model the spatial behaviour of pedestrians moving in group, the probability to move in one cell of the neighbourhood is firstly calculated based on the positions of the other agents of the group. In this model we refer to two types of group, denoted as simple and structured. As stated before, the distinction is due to the size: large groups are characterized by light cohesion, and they naturally split into subgroups of the simple type where the cohesion is stronger. A structured group is a group that can be composed of both simple groups and individual agents. The two functions $C(c)$ and $I(c)$ inside $U(c)$ are then used to model different attractions for agents belonging to a simple group $G$ or a structured one $\bar{G}$, identifying respectively intra- and inter-group relationships:

$$
\begin{gathered}
C(c)=\left[\left(\eta \cdot \sum_{\hat{a} \in G \backslash\{a\}} \frac{\left\|\vec{x}_{a}-\vec{x}_{\hat{a}}\right\|-\left\|\vec{x}_{c}-\vec{x}_{\hat{a}}\right\|}{|G|-1}\right) \cdot 2\right]-1 \\
I(c)=\left[\left(\eta \cdot \sum_{\hat{a} \in \bar{G} \backslash G} \frac{1}{\left\|\vec{x}_{c}-\vec{x}_{\hat{a}}\right\| \cdot|\bar{G} \backslash G|}\right) \cdot 2\right]-1
\end{gathered}
$$

where $\eta$ is a normalization factor that, together with final $\cdot 2$ and -1 , allows to translate the cohesion value into the range $[-1,1] .|G|$ defines the size of the group, while $\|\vec{x}\|$ denotes the 2 -norm of a vector $\vec{x}$. By its definition, the function $C(c)$ brings to much more different values for the neighbourhood of the 
agent and, thus, to a more determined behaviour of agents belonging to a simple group. The attraction between two agents belonging to different simple groups, both grouped in the same structured one, decreases in a inverse proportional way with respect to their distance (Eq. 6). Note that, in this equation, $G=\{a\}$ if an agent $a \in \bar{G}$ but it does not belong to a simple one. In addition, while the Eq. 6] is not defined for the case of distance equal to $0 \mathrm{~m}$ (i.e. the agents are overlapping), this cannot happen during the simulation because: (i) the utility is computed only for cells where the movement is possible and (ii) the overlapping between agents of the same group is not allowed.

The introduction of two distinct functions for the attraction inside simple and structured groups is aimed at providing an evident differentiation of behaviour: while agents of the same simple group must preserve a relative cohesion during the simulation by assumption, an agent can be effectively influenced by another one of the same structured group only at a relatively close distance (depending also to the parameter $\kappa_{i}$ ), due to the inverse proportional nature of Eq. 6. With this difference we want to provide a way to the user for modelling large groups as schoolboys or team supporters, where a gregarious behaviour is clearly observable but, due to the dimensions of these groups, each member is only influenced by his/her close companions. The function described in Eq. 5 instead is unaltered by the distance of the other simple group members (although there is a limit in the perception capabilities of each agent), because it is aimed to model couples, friends or family members that shall not permanently split during the simulation.

\subsubsection{Moving towards the same direction}

While the idea of structured groups will not be used for the tests discussed in this paper, the function $I(c)$ is as well used to generate following behaviour in situations of counter-flows. In this case, the group $\bar{G}$ is virtual and it is composed by agents perceived by the evaluating one, which are following the same target and whose positions are closer to it. To limit the burden of calculations, the set 


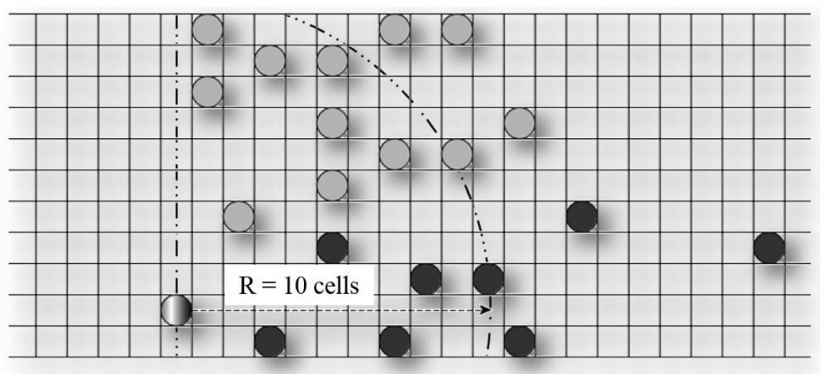

Figure 3: Perception of counterflow situation by the evaluating agents ahead moving in the same direction (dark grey) and counterflow ones (light grey). The group $\bar{G}$ of agents to follow is composed by grey agents inside the delimited area.

is limited to agents in a surrounding ${ }^{3}$ of about $4 \mathrm{~m}$.

This rule of the dynamics is graphically explained in Fig. 3. While the agent $\hat{a}$ perceives that in front of it there are other agents moving in opposite direction (i.e. directed toward $T_{b}$ ), the following behaviour is activated by composing an ad hoc structured group $\bar{G}$ for $\hat{a}$ with agents within $4 \mathrm{~m}$ of distance, which are located ahead of him and are moving towards the same direction. This leads to the formation of stable lanes in situation of counter-flow even at moderate densities. While this is slightly different from other approaches proposed in the literature to achieve lane formation without necessarily impose a gregarious behaviour (see, e.g., 45]), for the time being the usage of this already available mechanism has been chosen for sake of simplicity. Some ongoing works by the authors are already aimed to investigate this particular phenomenon and its modelling by means of a more accurate consideration of the pedestrian personal space.

\footnotetext{
${ }^{3}$ The set of agents in a fixed surrounding can be composed in an efficient way by means of a coarser grid used to index positions of agents.
} 


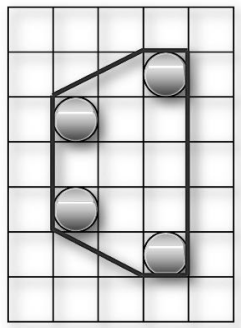

(a)

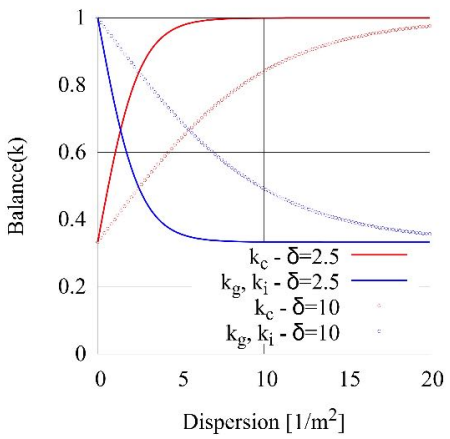

(b)

Figure 4: (a) Convex polygon describing the area occupied by the group and referred to calculate its dispersion. (b) Example of values of the function Balance for the different calibration weights, configuring different values of $\delta$.

\subsubsection{Staying together}

By acting on the parameters of $U(c)$ during the simulation it is possible to describe different states of the same pedestrian in different moments of a single simulated scenario. As already explained in [4, this strategy is applied to allow group members to adapt their behaviour in dense situations or in presence of obstacles. According to this method, weights $\kappa_{g}, \kappa_{c}, \kappa_{i}$ are varied and possibly inhibited according to an index that describes the dispersion of the group.

Quantifying such feature is a first issue in this context and for the proposed model we use an algorithm that calculates the area of the convex polygon build based on positions of group members. It must be noted that using only positions of agents lead to an different result for the case of dyads, because the algorithm actually produces a segment instead of a polygon. Hence, for all cases we extend the input points of the algorithm to the corners of cells where agents are located, as shown in Fig. 4(a). Furthermore, the space occupation of pedestrians is also considered in this way. Finally, the dispersion of a group $G$ is calculated as:

$$
\operatorname{Dispersion}(G)=\frac{\text { Area }(\text { ConvexPolygon }(G))}{|G|}
$$


The value of Dispersion is calculated by members of a group $G$ every timestep of the simulation, and their local values of $\kappa_{g}, \kappa_{c}, \kappa_{i}$ are modified according to the function Balance:

$$
\text { Balance }(\kappa, G)= \begin{cases}\frac{\kappa}{3}+\left(\frac{2 \kappa}{3} \text { DispersionBalance }(G)\right) & \text { if } \kappa=\kappa_{c} \\ \frac{\kappa}{3}+\left(\frac{2 \kappa}{3}(1-\operatorname{DispersionBalance}(G))\right) & \text { if } \kappa=\kappa_{g} \vee \kappa=\kappa_{i} \\ \kappa & \text { otherwise }\end{cases}
$$

$$
\text { DispersionBalance }(G)=\tanh \left(\frac{\operatorname{Dispersion}(G)}{\delta}\right)
$$

where $\delta$ is another parameter that, together with $\kappa_{c}$, acts on the behaviour of agents walking in groups: the higher its value, the lighter the effect of the dispersion on the maintenance of the cohesion. In particular, the usage of tanh in Eq. 9 provides a non-linearly increasing value in the range $[0,1]$ describing if the group is fragmented. In Eq. 8 this value is used to intensify/inhibit the parameters $\kappa$ related to the attractive components of the utility.

The configuration of these two values is thus determinant for the spatial behaviour of agents in groups, and the respective calibration will be subject of study in the following section.

\subsubsection{Avoiding modelling artifacts}

Although intuitively the above described method for preserving cohesion of a group can produce the expected results (and it is basically the mechanism adopted in our previous modelling efforts of group influence in pedestrian simulation), no particular attention is still given to shape preservation and to avoiding modelling artifacts that can be generated by this attempt. We discuss now two issues that can arise in particular when considering dyads, in which a typical shape (especially in low density situations) is the line abreast pattern [3] (i.e. pedestrians walking towards the same direction side by side, not far away from each other but not even walking attached one to the other). 


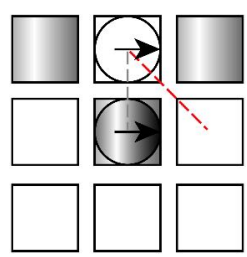

(a)

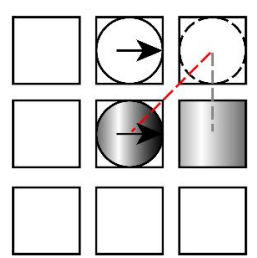

(b)

Figure 5: Methods for the simulation of the cohesion of simple groups: the previous method (a) considers distances from the current position of the other member, leading to have higher $C(c)$ values for cells $c$ in its direct surrounding (highlighted ones). In the proposed method (b) the position is predicted using the previous direction of movement, leading to an increased utility for the forward cell.

Avoiding implausible slowdowns - Equation 5 indicates which is the most proper cell to move considering the cohesion within simple groups. The evaluation of potential future cells privileges positions minimising the distance from other members, considering their current position. This mechanism is capable of preserving the cohesion and it already led to good results in terms of aggregated data about group dispersion during the simulation (see the results in [5]). Nonetheless, more detailed tests aimed at evaluating the spatial pattern of simple groups have emphasized some side effects of this formula in case of grouped agents walking side-by-side. Figure 5(a) graphically exemplifies how the current formulation of the model can lead to sudden stops of group members not motivated by upcoming obstacles or potential conflicts with other pedestrians: the agent uses the distance between the neighbour cell and the group member (dashed red line in the left figure) as the information to evaluate the cohesion, but this represents a reasonable choice only under the assumption of a non-movement action for the other companion. This means that the "stand still" choice utility is slightly emphasized: although this choice is still generally not the most favored one, since the overall utility function also considers the goal orientation $G(c)$, statistically this contribution will eventually cause an increase of sudden stops of pedestrians that are members of groups, which 
is at the same time negative for aggregated measurements and also leading to microscopic implausible artifacts in generated trajectories.

The formulated solution to this issue represents one of the novel steps proposed in this paper and it implements the concept of prediction in the calculation of the future distance:

$$
C(c)=\left[\left(\eta \cdot \sum_{\hat{a} \in G \backslash\{a\}} \frac{\left\|\vec{x}_{a}-\vec{x}_{\hat{a}}^{\bullet}\right\|-\left\|\vec{x}_{c}-\vec{x}_{\hat{a}}^{\bullet}\right\|}{|G|-1}\right) \cdot 2\right]-1
$$

Where the predicted position $\vec{x}_{\hat{a}}^{\bullet}$ is calculated using the velocity vector $\vec{\nu}_{\hat{a}}$ of the agent $\hat{a}$ (Fig. $[5(\mathrm{~b})$, describing previous movement direction:

$$
\vec{x}_{\hat{a}}^{\bullet}=\vec{x}_{a}+\vec{\nu}_{a}
$$

Avoiding not plausible conflicts - As it has been explained in Sec. 3.2 similar choice of movement of agents at a given step can be inhibited by the conflicts management rules, in order to preserve the basic physics of the system. Groups of agents, on the other hand, must be considered as an exception to these rules, since their walking close to each other can potentially lead to very frequent conflicts that are not realistic since members of groups actually continuously regulate their mutual position to preserve the possibility to verbally and nonverbally communicate. Not considering this aspect also leads to excessive drops in the walking speed of members of groups at low densities.

Figure 6, on the left, exemplifies this issue by showing a case where two grouped agents chose the same destination cell, which is also the most probable one in that situation. The issue is solved by introducing a rule for the management of conflicts where only members of the group are involved: these situations are managed by changing the destination cell of one (or more) of the members to other free cells of the Von Neumann neighbourhood of the cell in which the conflict is originated. The management of the conflict shown in Figure 6 leads to the uppermost agent moving forward instead of diagonally, as a consequence of a micro-level coordination with the other group member. In case of multiple 

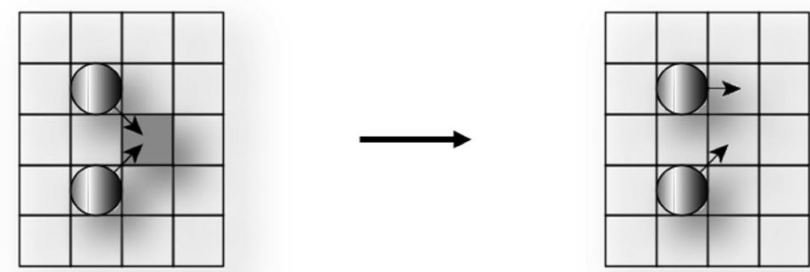

Figure 6: Example of a conflict situation involving two grouped agents (left) and the outcome given by the updated conflict management rules.

available possibilities for solving the conflict, cells closer to the target are preferred (the rationale is that the coordination does not only solve conflicts, but it favors most rational solutions).

It must be noted that this additional set of rules, together with the new formulation of $C(c)$ as in Eq. 10 might lead to an error in the opposite direction, i.e., inhibiting the capability of the model to reproduce a relevant difference in walking speed as effect of the cohesion mechanism. As it will be shown in the results of Sec 4.3 , at low densities the cohesion mechanism still provides a sensibly lower velocity of group members, now smoother with respect to the previous definition of the model.

\subsection{Limitations}

The present approach and model presents two main classes of limitations: a part of them is due to the intrinsically discrete modelling approach, whereas other ones are more closely related to the specific mechanisms defined in our way of managing dyads.

First of all, discrete modelling approaches present intrinsic limitations in the spatial representation: pedestrians are generally considered as positioned within a cell (in some cases in the centre of the cell, for other models in any point of a cell), and their movement is necessarily atomic, from a cell to another. This 
implies that generated trajectories are necessarily "jagged" and they are not immediately $\left.\right|^{4}$ suited to generate a smooth visual representation of pedestrians' movements. Nonetheless, if the aim of the simulation is to achieve more coarse grained indicators, such as average/maximum travel times (and related standard deviations), space utilisation maps (heat maps describing the aggregate usage of space by pedestrians), flows measured in specific sections, the achieved results can be quite similar to continuous modeling efforts and sometimes requiring lower computation times (see, e.g., [46] for a comparison among commercial continuous and discrete simulation tools).

With specific respect to group modelling, the discrete approach presents a difficulty in rendering the fine grained interpersonal distance regulation by group members. Results of a long term observation and analysis described in [7, to mention one of the most detailed analysis of this phenomenon, show that (at least within that specific cultural context, that is, a Japanese multipurpose building including a commercial area - for which the authors later provided an even richer and more detailed analysis in 8] interpersonal distances among members of dyads are not always compatible with a discrete modelling effort: across the observed densities, which vary between almost zero and 0.3 pedestrians per square metre, in most situations dyad members are walking at a distance between 47 and $65 \mathrm{~cm}$. Within a discrete modelling approach this value could be either 40 or $80 \mathrm{~cm}$ (in case of a discretization in $40 \mathrm{~cm}$ sided cells), so it is basically a matter of choosing the mutual position that minimise the error, which cannot be avoided.

Moreover, we do not have available a dataset indicating how do members of dyads having different desired velocities manage their interpersonal distance regulation and overall choice of the actual velocity. A reasonable conjecture is that members of a dyad actually adjust their desired speed to reach a consensus

\footnotetext{
${ }^{4}$ Processes of interpolation of discrete positions assumed by pedestrians in different timesteps might produce a visually more pleasant effect, although the actual validity of this kind of trajectory would call for additional analyses.
} 
value: for instance, a younger and taller pedestrian might slow down to be able to walk side by side with a shorter and older friend or relative. Nonetheless, we must say that we do not have, as of this moment, a way of validating this conjecture and the mechanism of dyads interpersonal distance regulation in case of heterogeneous desired speeds for group members.

Finally, the adopted mechanisms could likely be generalised for managing small groups but larger than a dyad (i.e. groups of three or four members). Nonetheless, the overall calibration procedure and generally validation tests would imply an additional effort and additional empirical data not available at this moment, so it is object of future works.

Results shown and discussed in the following Section will also provide additional considerations on these points.

\section{Calibration and Validation Tests}

For the purpose of discussing and validating the dynamics simulated by the model, this Section will provide several simulation batches aimed at, respectively:

- calibrating the components dedicated to the behaviour of pedestrians in group;

- validating the overall model with the fundamental diagram and verifying the aggregated effects of the presence of groups;

- verifying the dynamics of individual and dyads at low densities simulated with the calibrated model;

- verifying the trajectories of individual and dyads in more complex geometries and environments.

The batches will share many calibration parameters of the model, most of them resulting from previous exploration and validation works (see [4] and [32]). An important parameter, fundamental for the simulated dynamics and also 


\begin{tabular}{|c|c|c|}
\hline Parameter Name & Value & $\begin{array}{l}\text { Varied in } \\
\text { batch } \mathbf{n} \text {. }\end{array}$ \\
\hline \multicolumn{3}{|c|}{ Global Parameters } \\
\hline $\begin{array}{l}\text { time-step duration } \\
\text { cell side } \\
\text { desired speed of agents } \nu_{d} \\
\text { max speed of agents } \nu_{m} \\
\text { reaction time of agents } \xi\end{array}$ & $\begin{array}{l}0.25 \mathrm{~s} \\
0.4 \mathrm{~m} \\
1.6 \mathrm{~m} / \mathrm{s} \\
1.6 \mathrm{~m} / \mathrm{s} \\
0.5 \mathrm{~s}\end{array}$ & $\begin{array}{l}- \\
- \\
- \\
-\end{array}$ \\
\hline \multicolumn{3}{|c|}{ Decision Parameters } \\
\hline $\begin{array}{l}\text { utility parameter } \kappa_{g} \\
\text { utility parameter } \kappa_{o b} \\
\text { utility parameter } \kappa_{s} \\
\text { utility parameter } \kappa_{d} \\
\text { utility parameter } \kappa_{o v} \\
\text { utility parameter } \kappa_{i} \text { for } \\
\text { the lane formation }\end{array}$ & $\begin{array}{l}8.0 \\
4.0 \\
28.0 \\
2.0 \\
2.0 \\
6.0\end{array}$ & $\begin{array}{l}- \\
- \\
- \\
2 \\
2\end{array}$ \\
\hline \multicolumn{3}{|c|}{ Group Cohesion Parameters } \\
\hline $\begin{array}{l}\text { utility parameter } \kappa_{c} \\
\text { parameter } \delta \text { of Eq. } 9\end{array}$ & $\begin{array}{l}15.0 \\
5.0\end{array}$ & $\begin{array}{l}1 \\
1\end{array}$ \\
\hline \multicolumn{3}{|c|}{ Conflict Resolution Parameters } \\
\hline $\begin{array}{l}\text { fric }_{l} \\
\text { fric }_{h}\end{array}$ & $\begin{array}{l}0.8 \\
0.96\end{array}$ & - \\
\hline
\end{tabular}

Table 1: Calibration Parameters used in the simulation batches.

related to one of the novel aspect of this work, is the reaction time, which in all simulation is set to $0.5 \mathrm{~s}$, in order to reproduce the fundamental diagrams shown in Sec. 4.2. Table 1 describes all the parameters values and which ones are varied in the three batches.

\subsection{Calibration of group behaviour with empirical data}

The behaviour of agents in groups is calibrated using experimental data from a controlled experiment performed in 2015 in a corridor setting. A full description of this experiment can be found in [6]. In this section we briefly describe the two experimental procedures which are reproduced with the simulation model.

The setting of the experiment was represented by a long corridor of about $38 \times 3 \mathrm{~m}^{2}$, where the 54 total participants were initially distributed among the two drawn grids of $12 \times 3 \mathrm{~m}^{2}$, composed of 6 rows $\times 9$ columns and located at the two extremes of the environment. The dynamics in a measurement area of 

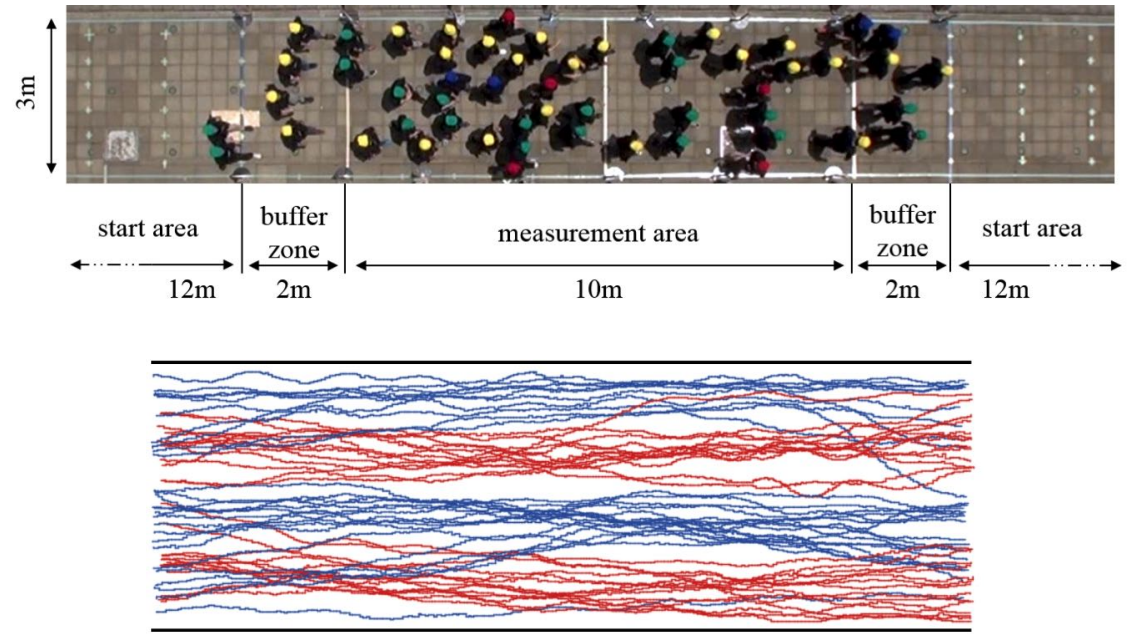

Figure 7: Top: frame from a video of the experiment involving dyads [6], with a logical description of the setting. Bottom: sample trajectories inside the measurement area of one iteration of the 3-3 procedure (colors are assigned to identify the direction of flows).

$10 \times 3 \mathrm{~m}^{2}$, at the center of the environment, was recorded with a camera placed at a relatively high position. Thanks also to coloured hats wore by participants, it was possible to automatically and precisely track their positions using the software PeTrack [4]. A graphical description of the setting and an example result of the tracking are shown in Fig. 7 .

According to the experimental procedures (which were 4 in total), the counterflow condition at the measurement area was controlled: from uni-directional flow (6-0, 6 participants per column in one starting grid) to perfectly balanced bi-directional flow (3-3, 3 per column in both starting grid). Also, we experimented the effect of groups of 2 persons (dyads) by randomly selecting pair of participants and asking them to stay close as they were friends while walking. The proportion individuals / group members in all procedures was respectively of $30 / 24$. Each procedure was repeated 4 times to gather more data.

Regarding the simulation, we hereby analyse the plausibility of the behaviour of simulated dyads, which in the model can be regulated by the two parameters $\delta$ and $\kappa_{c}$ acting on Eq. 3 and Eq. 9 respectively. For the purpose of this calibration 
work we will analyse and compare the several data for both the experiment and simulation runs:

- average speeds of individual participants;

- average speeds of coupled participants;

- distributions of relative positions of dyads, which describe their average spatial pattern assumed during the procedure.

Due to the stochastic nature of the model, we performed 24 simulation iterations for each configuration (a parallel batch of 3 sequential runs in a machine with 8 cores) of the two parameters, exploring a square range defined as

$\left\{\left(\kappa_{c}, \delta\right) \in \mathbb{N}^{2}: \kappa_{c}, \delta \leq 30\right\}$. The quantity of iterations was actually enough to achieve stable results and the range was sufficient to recognize the trend and identify possible good calibration values. Moreover, we made the following assumptions to configure the simulations: (i) every pedestrian is configured with a desired speed of $1.4 \mathrm{~m} / \mathrm{s}$, able to describe the average speed of young people [2] and also to reproduce simulated mean speeds in range of observed data in the two experiments; (ii) all pedestrians are generated in initial positions that reflect the grids of the experiment, also granting a certain non determinism and variability (i.e., pedestrian's position within the column is randomly chosen); (iii) a similar environment and measurement area is depicted in the simulation. The error metrics employed to evaluate the difference between observations and simulations will be now discussed, together with their results.

\subsubsection{Spatial error for dyads}

This metric compares the distributions of relative positions of dyads, with respect to their centroid. In particular, the centroid of a group at a certain frame is simply calculated as the average between positions of its members. For all frames of the video, relative positions are then collected and a discrete distribution of points located inside cells of $0.2 \times 0.2$ is calculated. Results of the experiment and simulations (the latter with the chosen value of $\left(\kappa_{c}, \delta\right)$ 
and related to the case of procedure 3-3) are shown in Fig. 8 (a) and (b). The square range of side $2 \mathrm{~m}$ is enough to contain all observed and simulated points in these scenarios. The granularity of the graphs is different for the experimental observation (in which the actual phenomenon is continuous in nature, but analysed with a fine grained grid of $5 \mathrm{~cm}$ sided cells) and for the simulation (that is discrete, with $40 \mathrm{~cm}$ sided cells), but we preferred to show the experimental data with the highest possible level of detail. It must however be noted that the most frequently occupied cell in the simulation side is actually associated to a higher number of cells of the observation side, whose frequencies should be summed up to achieve a fair comparison. Unlike the comparison with the naturalistic observation described in the already cited [7], the data acquired by means of pedestrian experiments (gathered in an artificial situation characterised by locally high levels of density) are more closely reproduced by the simulations: this is very likely due to the overall higher densities which pushed the participants to stay closer to each other. We will show later on that in much lower density situations the spatial distribution generated by the model are less in tune with experimental data (again from [7]).

Given the discrete distributions of both observations $\left(\Phi_{o b s}\right)$ and simulations $\left(\Phi_{\text {sim }}\right)$, the spatial error is then defined as the mean absolute error (MAE) for all points in the range of interest:

$$
\text { error }_{s p}=\frac{\sum_{(x, y)}\left|\Phi_{\text {sim }}(x, y)-\Phi_{o b s}(x, y)\right|}{2}
$$

Figures 8 (c) and (d) shows the heatmaps of MAE value for the procedure 6-0 and 3-3. The error varies in a quite large range, but it is possible to identify a trend of pairs $\left(\kappa_{c}, \delta\right)$ such that the error drops between 0.2 and 0.3 . Even if overall these errors appear to be high, the simulated distribution of relative positions in Fig. 8 (b) shows that the model has respected the most frequent spatial pattern of dyads, indicating that they walked side-by-side during most of the simulation time. 


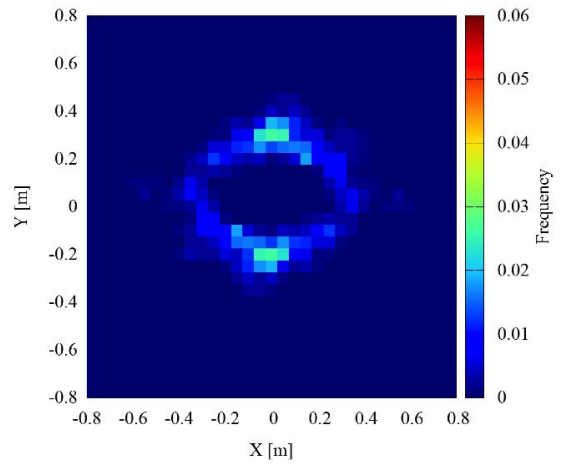

(a)

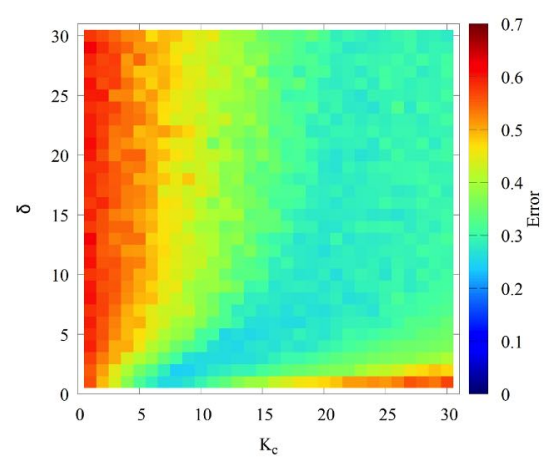

(c)

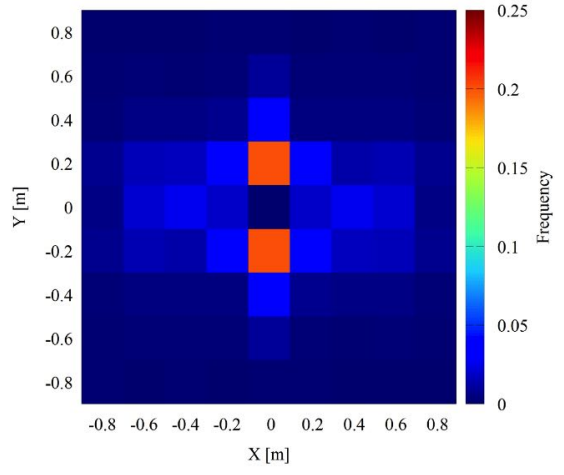

(b)

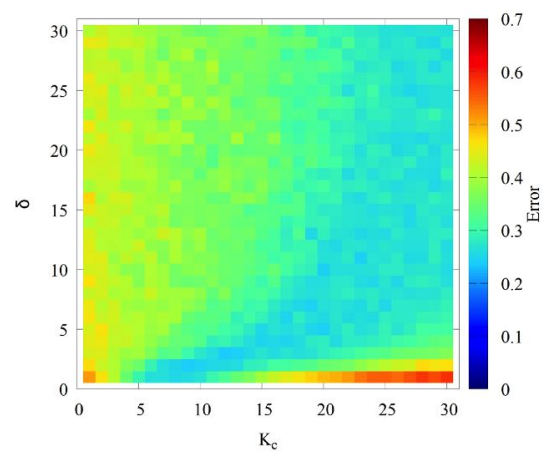

(d)

Figure 8: Observed (a) and simulated (b) distribution of relative positions of dyads, for the case of the 3-3 procedure. On the bottom, the matrix describing the MAE for the case of 6-0 (c) and 3-3 procedures (d) in the explored range of parameters.

\subsubsection{Error on resulting speeds of pedestrians}

This metric is related to the evaluation of the speeds of individuals and dyads. Since the number of dyads and individuals differs, the absolute error resulting in the two separate cases is unified with a weighted mean based on the ratio of individuals $\left(\frac{|\mathbf{I}|}{|\mathbf{T}|}=\iota\right)$ and dyad members $\left(\frac{|\mathbf{\Delta}|}{|\mathbf{T}|}=\chi\right)$ on the total population. In addition, the error on each component is normalized according to the average value of the observation. Thus the speed error related to one 


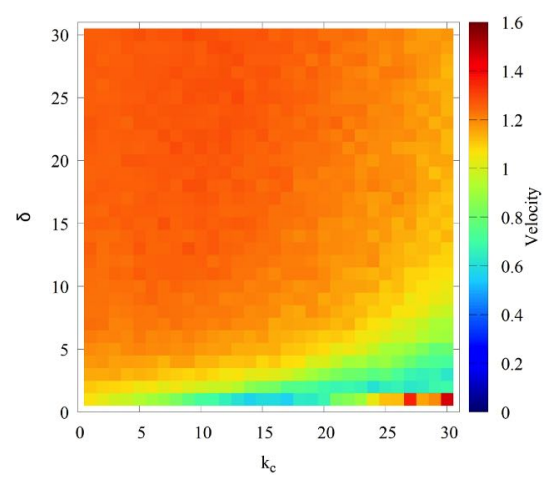

(a)

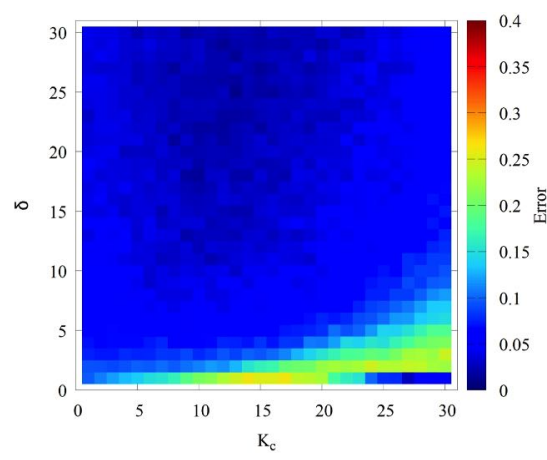

(c)

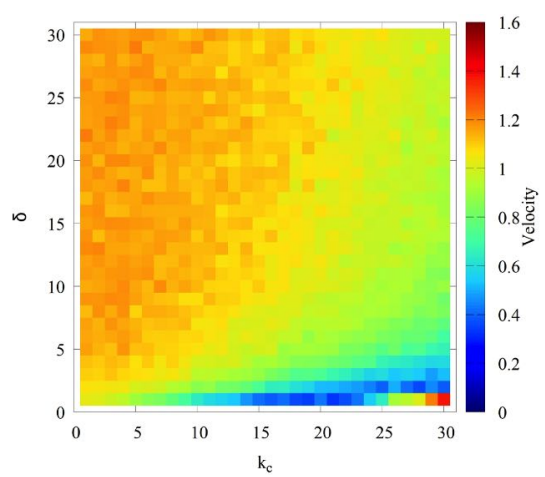

(b)

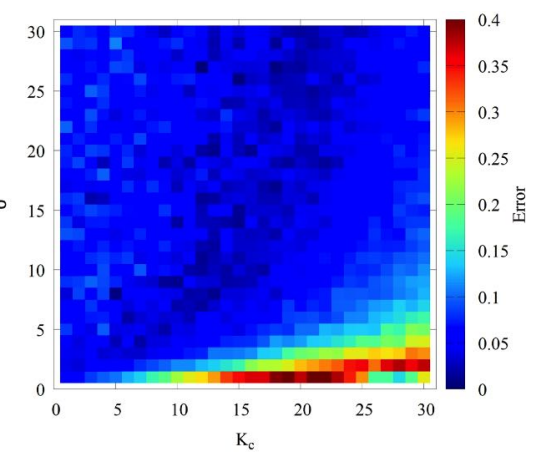

(d)

Figure 9: On the top: average speeds of dyads in the simulation of procedure 6-0 (a) and 3-3 (b) at the variation of $\left(\kappa_{c}, \delta\right)$. On the bottom: values of error $_{\nu}$ for the two procedures (the order of presentation is the same).

experimental procedure is defined as:

$$
\begin{aligned}
\operatorname{error}_{\nu} & =\iota \cdot \frac{\left|\mu_{a \in \mathbf{I}}\left(\left\|\overrightarrow{\nu_{a}}\right\|\right)_{\text {sim }}-\mu_{a \in \mathbf{I}}\left(\left\|\overrightarrow{\nu_{a}}\right\|\right)_{o b s}\right|}{\mu_{a \in \mathbf{I}}\left(\left\|\overrightarrow{\nu_{a}}\right\|\right)_{o b s}}+ \\
& +\chi \cdot \frac{\left|\mu_{a \in \boldsymbol{\Delta}}\left(\left\|\overrightarrow{\nu_{a}}\right\|\right)_{\text {sim }}-\mu_{a \in \boldsymbol{\Delta}}\left(\left\|\overrightarrow{\nu_{a}}\right\|\right)_{o b s}\right|}{\mu_{a \in \boldsymbol{\Delta}}\left(\left\|\overrightarrow{\nu_{a}}\right\|\right)_{\text {obs }}}
\end{aligned}
$$

where $\mu_{a}\left(\left\|\overrightarrow{\nu_{a}}\right\|\right)$ is the average speed of the pedestrian $a$, either single $(\in \mathbf{I})$ or member of a dyad $(\in \boldsymbol{\Delta})$, on all frames of the video or of the simulation and while inside the measurement area. 
Results of this evaluation are shown in Fig. 9. Figures (a) and (b) describe the error on the speeds of dyad $5^{5}$ It is noticeable that the 3-3 procedure leads to a less stable dynamics at the measurement area, providing a higher variation of average speeds over the ranges of the two parameters. In particular, the effects of the two parameters is particularly understandable in these pictures: at the increasing of the $\kappa_{c}$ the effect of cohesion becomes more and more strong but and it negatively affects the speeds; on the other hand, $\delta$ acts by increasing the length of the transition between the phases where the group is considered compact or dispersed, thus relaxing the rule.

Despite the extremes of the heat maps, values are similar to the observed ones: in the procedure $6-0$, the average speed of both individuals and dyads was observed as about $1.3 \mathrm{~m} / \mathrm{s}$, while in procedure $3-3$ of about $1.2 \mathrm{~m} / \mathrm{s}$ for individuals and $1.1 \mathrm{~m} / \mathrm{s}$ for dyad members. By looking at Fig. 9 (c) and (d), it is possible to recognize a range of values for the pair of parameters $\left(\kappa_{c}, \delta\right)$ providing low errors for both procedures, highlighting that the model is able to fit these observation quite well.

\subsubsection{Aggregating errors}

After having computed the matrices of error for both speed and position distribution, it is possible to provide a unified analysis to understand the optimal configuration of parameters for these data. Firstly, the aggregated analysis for a given dataset (i.e., procedure) is simply defined as the average between the two errors:

$$
\text { error }_{\text {avg }}=\frac{\text { error }_{s p}+\text { error }_{\nu}}{2}
$$

Results are shown in Fig. 10 (a) and (b) for both procedures. Finally, all results are summarized by computing again the average for the two datasets, defining a unique index allowing to choose an optimal calibration. As shown

\footnotetext{
${ }^{5}$ for sake of space the ones of individuals are not reported, also since it was not much affected by the two calibration parameters.
} 


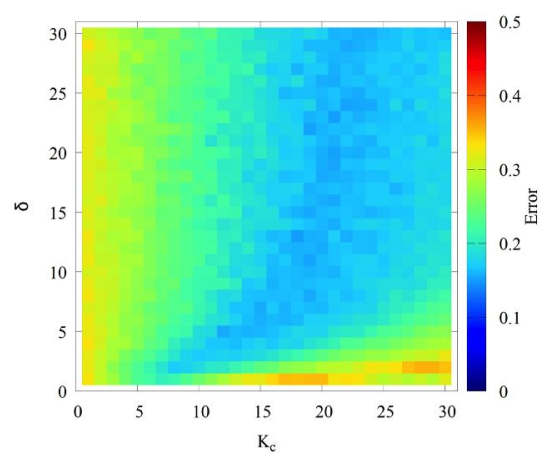

(a)

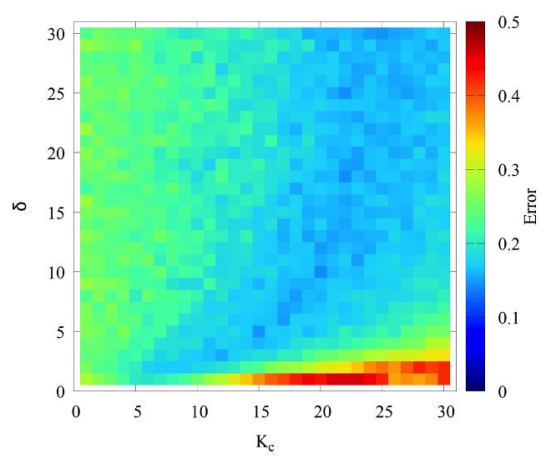

(b)

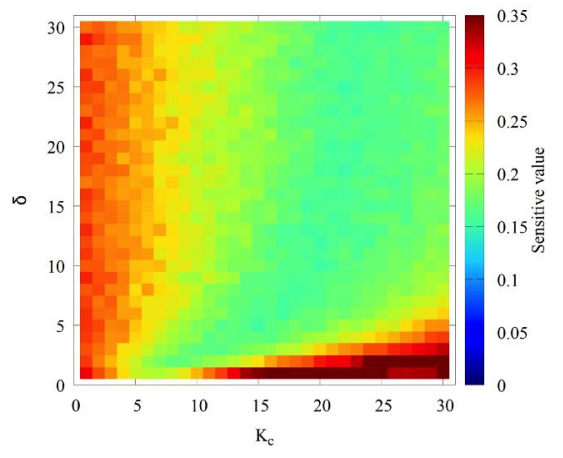

(c)

Figure 10: Average error values for the procedure 6-0 (a), 3-3 (b) and the final average of both (c). Note that in (c) the range of values was restricted to emphasize their differences.

with the previous analysis, there is no best option of parameters providing a minimum error, yet minimal values are distributed in the explored range with a particular trend. By assumption and to avoid problems in numerical computations, we then preferred lower values of parameters if they provide similar results. By looking at Fig. 10 (c), we thus chose the final configuration of $\left(\kappa_{c}, \delta\right)=(15,5)$. 


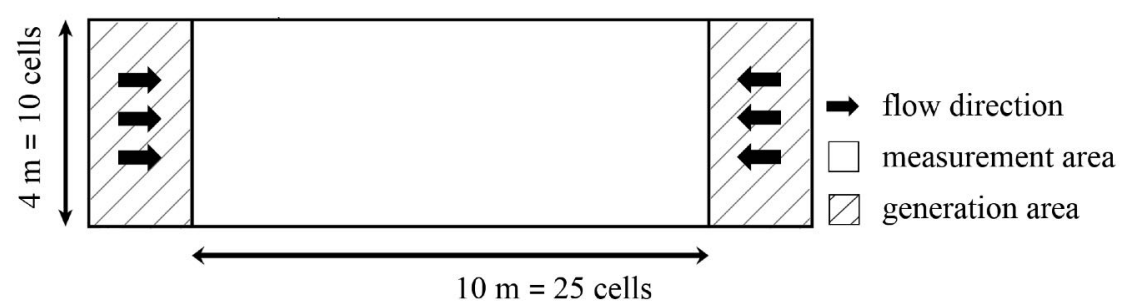

Figure 11: Logic representation of the corridor setting. For the case of uni-directional flow, the generation area on the right is disabled.

\subsection{Analysis of the Fundamental Diagram}

The fundamental diagram is the primary instrument used for the validation of the physics reproduced by models of this kind. It describes relations between pedestrian density, speed and specific flow. In this section, the movement of individuals and dyads is investigated in order to explore and analyse the performance of dyads with respect to generated fundamental diagram data.

The simulation batch here presented is run in a unique setting describing a corridor of $10 \times 4 \mathrm{~m}^{2}$ as depicted in Figure 11, which hosts either a uni-directional or bi-directional and equally balanced flow of pedestrians, denoted respectively as SIM-1.x and SIM-2.x. For both experiments we tested two agents generation profiles, in order to introduce a crowd composed of only individuals (SIM-x.1) and a crowd with half of the population coupled to simulate dyads (SIM-x.2). To generate the dynamics, two generation areas of $2 \times 4 \mathrm{~m}^{2}$ are located at the extremes of the corridor (only one is active for scenarios SIM-1.x), introducing pedestrians with a rather low frequency of 0.25 pers $/ \mathrm{s}$.

The central part of the environment is characterised by a measurement area used to compute the output: by collecting entrance $t_{1}$ and exit $t_{2}$ event times (in seconds) for every agent, the respective travel time $t t\left(t_{1}, t_{2}\right)=t_{2}-t_{1}$ is calculated and used to compute the average speed $\nu\left(t_{1}, t_{2}\right)=\frac{10}{t t\left(t_{1}, t_{2}\right)} \mathrm{m} / \mathrm{s}$ to be associated with the density $\rho\left(t_{1}, t_{2}\right)$. This last value describes the mean number of pedestrians inside the measurement area in the time window $\left[t_{1}, t_{2}\right]$. In this way a reference global density $\hat{\rho}_{i}$ is configured for each simulation $i$ of the batch, 


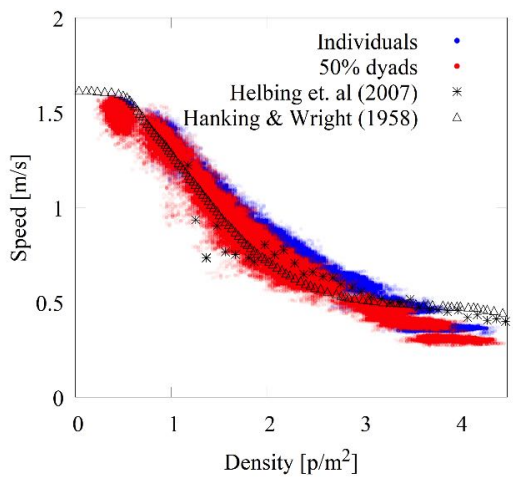

(a)

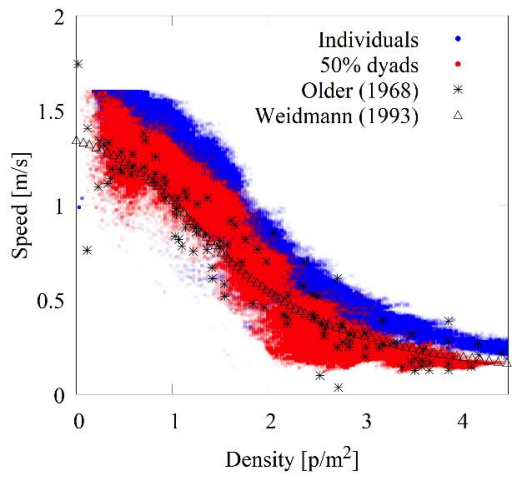

(c)

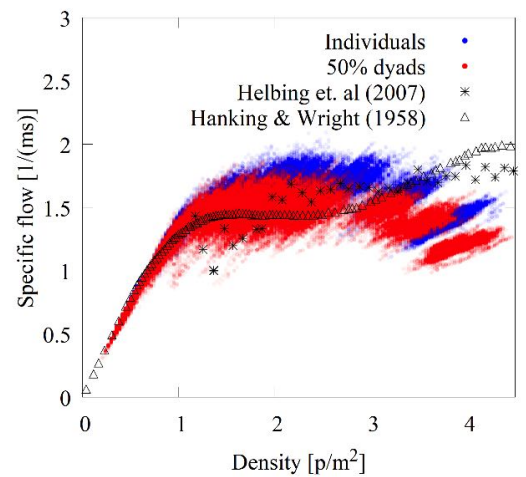

(b)

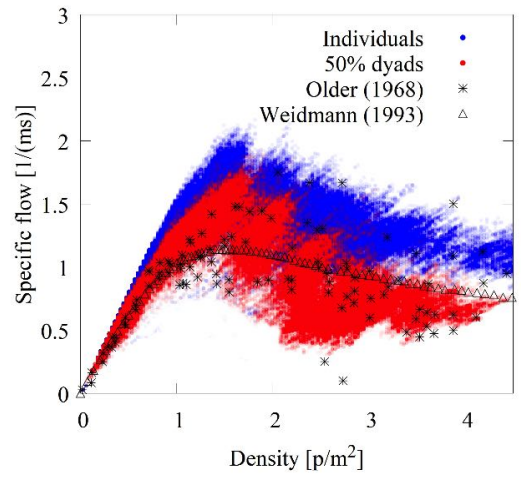

(d)

Figure 12: Density-speed and density-flow diagrams for the simulations of SIM-1.x $(\mathrm{a}-\mathrm{b})$ and SIM-2.x (c - d) scenarios, with literature datasets for comparison.

describing the maximum number of pedestrians in the environment, but each iteration will produce a set of data in a relatively close range of $\hat{\rho}_{i}$. To explore the fundamental diagram data, the set of simulated densities is defined with a tick of 0.5 pers $/ \mathrm{m}^{2}$, so that the configured global density $\hat{\rho}_{i}=0.25+0.5 \cdot i, i \in[0,8]$. The tick of 0.5 led to the achievement of little gaps between the clouds of points related to the highest experimented densities, particularly noticeable in the plot density-flow for the 1-directional case (Fig. 12(b)). On the other hand, the range 
and the trend of the results is already explained by the achieved datasets. Due to the stochastic nature of the model, we performed 24 runs for each configuration (3 runs per processor in a machine with 8 cores), which we found to be sufficient to achieve stable results.

The calibration of the model parameters is based on values achieved with previous studies (see [4] and [32]). In this simulation batch we mainly investigated the value of the parameter $\xi$ describing the reaction time of pedestrians and influencing their travel times in congested situations. Results shown in Figure 12 refer to the final calibration, with $\xi$ set as 0.5 s and other parameters configured as described in Table 1 at the beginning of this section. Note that the parameter $\kappa_{i}$-managing the attraction towards agents moving in the same direction- was set to a relatively high value to induce lane formation and avoid rapid phase transitions between free flow and gridlock in bi-directional cases. Results fit very well datasets of field-observations and guidelines from the literature used for the comparison, both for the trend and range of values. As expected, the influence of the $\xi$ is essentially to cause a decrease of the achieved flow in situations of medium (about 1 pedestrian per square metre) to relatively high densities (up to 3 pedestrians per square metre), making the overall point cloud less "linear" than the one we achieved in 32, especially in the 1-directional case. Finally, although we do not intend to present and discuss a thorough analysis of the efficiency of the proposed approach, we preserve the possibility to simulate agent populations faster than real-time up to about few hundreds of agents in a desktop class PC, although at this stage of the work we mainly aimed at achieving an effective reproduction of pedestrian and dyads behaviour, and optimizations leading to significant improvements are possible and object of future works.

\subsection{Individuals and Dyads at Low Densities}

The simulations here provided are aimed at analysing the capabilities of the model in reproducing the dynamics of individuals and groups in simple low

density situations. Two main scenarios representing free flow conditions are run 
in a long corridor of $40 \times 10 \mathrm{~m}^{2}$, simulating either (i) only individuals or (ii) only dyads. Moreover, we want to analyse the effects of varying the desired speed of agents on the results, hence we consider two configurations for this parameter which are checked independently: a baseline configuration of $1.6 \mathrm{~m} / \mathrm{s}$ (equal to the maximum speed) and a second one at $1.4 \mathrm{~m} / \mathrm{s}$. In all cases the environment is not configured as toroidal and the frequency of generation is set to $1 / 5 \mathrm{ped} / \mathrm{s}$ (1/10 dyads/s for the second simulation), with a total population of 200 agents.

Results of Fig. 13 (a) and (b) analyse the distribution of simulated speeds in the four simulations. The peak of the distribution for individual agents is higher to the one of dyads with both configurations of the desired speed. Moreover, it is visible that individual agents have mostly walked at their desired speed, highlighting the free flow condition, while dyads are slightly slowed down by the effect of cohesion. By comparing the figure we see that the difference gets smoother with a lower initial configuration of the desired speed.

By looking at the distributions of relative positions in Fig. 13 (c) and (d), we see that dyads mostly walk side-by-side in both cases. The configuration of a different desired speed does have an influence on the distribution: while with $1.6 \mathrm{~m} / \mathrm{s}$ the distribution reaches a value of about 0.28 in cells describing the line abreast pattern, this value decreases to about 0.23 in case of $1.4 \mathrm{~m} / \mathrm{s}$ in favour of cells describing a diagonal pattern of dyads. This limit is essentially caused by the probabilistic nature of the heterogeneous speed mechanism, which causes a member of the dyad to suddenly yield the position to reproduce its desired speed, not necessarily in sync with the other member of the dyad. Nonetheless, assuming a microscopic coordination among members of a dyad, this side effect could be easily overcome with a synchronous management of the desired speed for the group members, i.e., the activation for the movement at the beginning of the step (as explained in Sec. 3.2 This possible improvement will be subject of investigation with future works, and we wanted to present here a worst case scenario. 


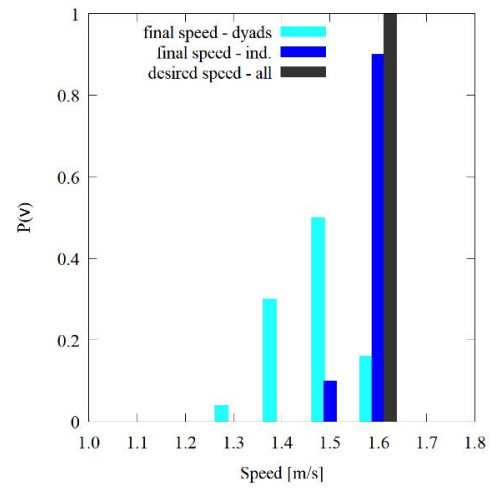

(a)

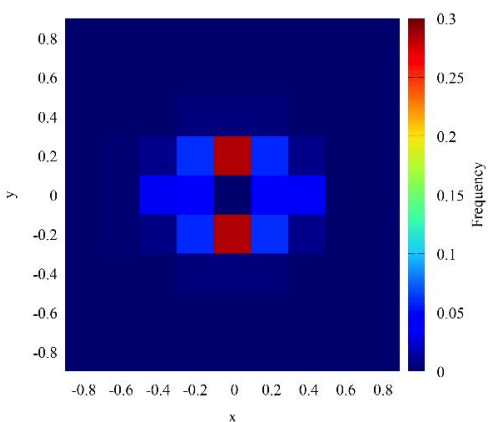

(c)

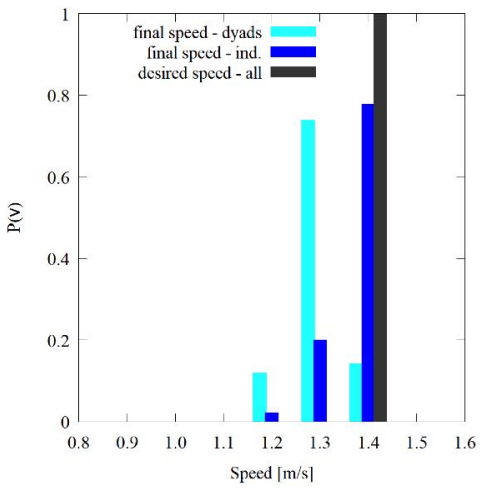

(b)

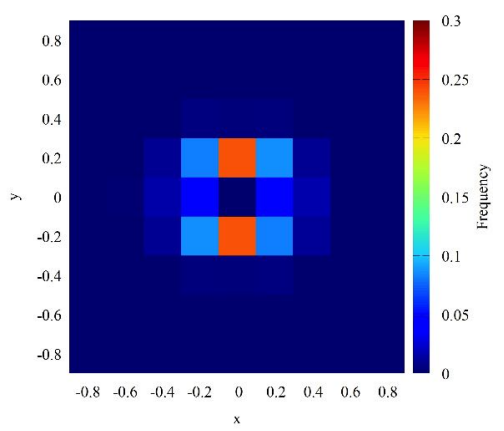

(d)

Figure 13: ( $\mathrm{a}$ - b) Distribution of simulated speeds of individuals and dyads in the simulation of the long corridor, with the $1.6 \mathrm{~m} / \mathrm{s}$ (a) and $1.4 \mathrm{~m} / \mathrm{s} \mathrm{(b)} \mathrm{configuration} \mathrm{of} \mathrm{the} \mathrm{desired} \mathrm{speed.}$ $(c-d)$ Distribution of relative positions of dyads (direction of movement towards right), with the $1.6 \mathrm{~m} / \mathrm{s} \mathrm{(c)} \mathrm{and} 1.4 \mathrm{~m} / \mathrm{s}(\mathrm{d})$ configuration.

\subsection{Qualitative Evaluation - Trajectories of Dyads}

To complement the above provided quantitative and qualitative evaluation of the model, we also describe the produced results by means of visualizations of the produced dynamics, to support the understanding of the behaviour of simulated dyads in terms of trajectories. The following renderings were generated by 

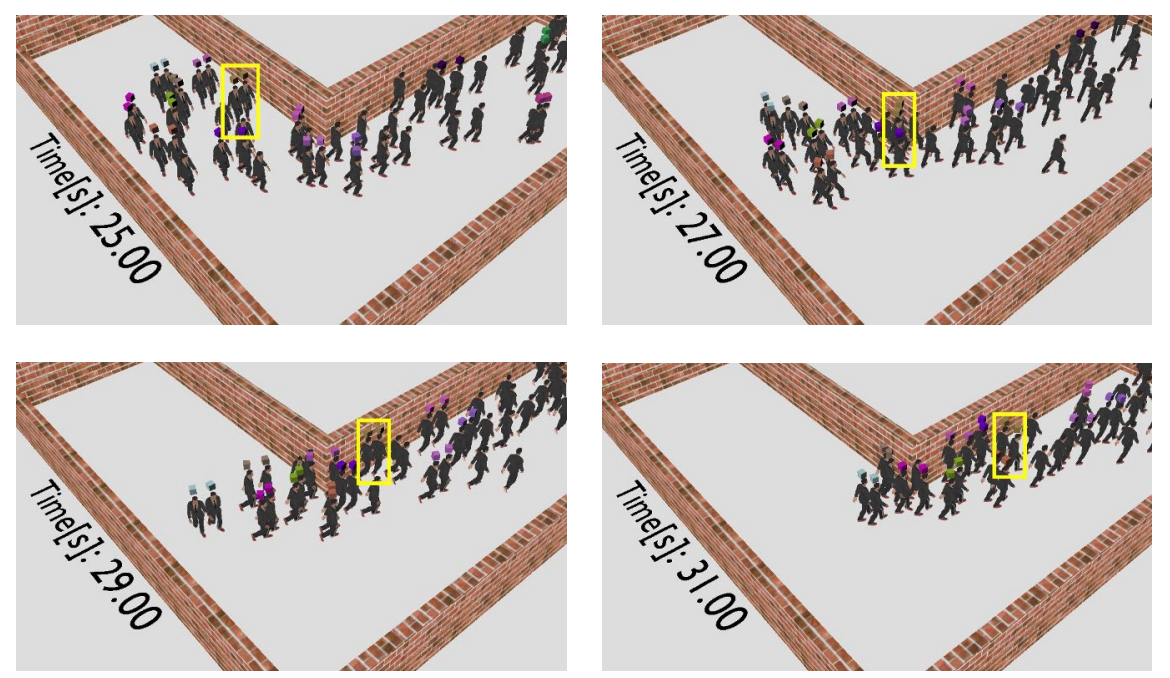

Figure 14: Screenshots of the simulation of the bended scenario.

means of a script enabling the Blender ${ }^{6} 3 \mathrm{D}$ modelling tool to import, analyse and render the discrete dynamics generated by the simulation system. The overall process of parsing, analysis, and rendering is rather simple and we will not provide a thorough description since it does not represent an improvement on the state of the art of pedestrian dynamics visualization systems. In a nutshell, the script parses two files, respectively associated to the discrete representation of the environment (described in Section 3.1) and to the trajectories generated by a single simulation. Walls and obstacles are represented as parallelepipeds occupying all not walkable space in the simulation. As assumption, each wall has a height of $2 \mathrm{~m}$. A unique animated human model is used to represent the position of each simulated pedestrian. Trajectories of pedestrians are rendered in a continuous way by interpolating instantaneous positions produced by the simulator, with a moving average of 5 steps $=1.25 \mathrm{~s}$. In this way, it is possible to achieve a smooth movement of agents without an excessive modification of the simulation result.

6 https://www.blender.org/ 

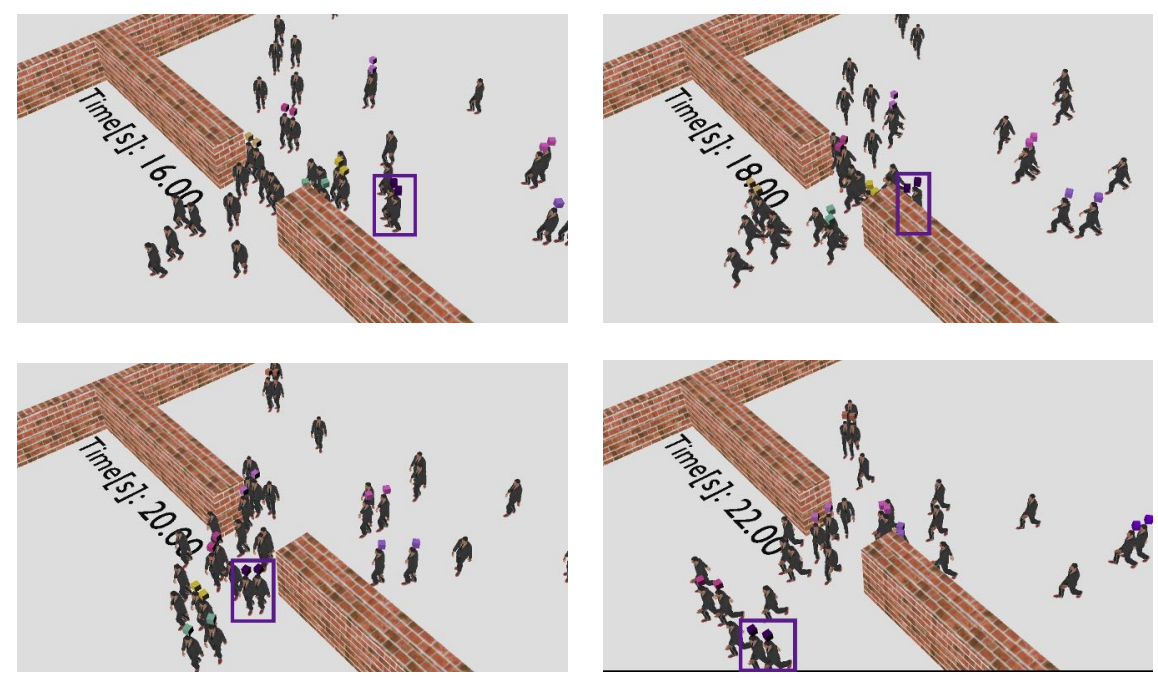

Figure 15: Simulation of the bottleneck scenario.

Figure 14 shows four renderings of a simulation associated to a 90-degrees bend crossed by a uni-directional flow of pedestrians. The population is composed of individuals and dyads in the same proportion; dyads are characterised by a coloured box on top of the head of members. The arrival rate of pedestrians is configured to ensure a consistent but not excessive flow, leading to a situation in which dyads can face both issues related to a change in the preferred direction as well as potential conflicts due to medium density.

The set of screenshots allows evaluating the overall space utilization and shapes of dyads: by looking at the highlighted dyad we firstly see that at time $25 \mathrm{~s}$ the two agents are approaching the bend by walking side-by-side, like most other dyads in the first part of the scenario. While they get closer to the bend, they change their shape into a river-like pattern (one in front of the other), but in the final screenshot (time $31 \mathrm{~s}$ ) they change back again to the line abreast pattern and they preserve this shape until they move out of the simulation.

The second environment represents two small rooms joined by a central bottleneck of width $3.2 \mathrm{~m}$, which is crossed by a uni-directional flow composed of individuals and dyads in equal proportion. In this scenario the bottleneck 
is wide enough to avoid the emergence of a relevant congestion in the scenario, yet the incoming flow is sufficient to produce noticeable slowing down for the agents. Pictures show that the highlighted pair is forced to change the side-byside pattern when approaching the bottleneck, but the cohesion mechanism of the model lets them maintain a close distance even in the congested zone, and it also allows them to get back to a line abreast pattern once the surrounding condition returns to a free flow state.

\section{Conclusions and future works}

This paper has presented a model for the flexible management of group cohesion within pedestrian simulation; the model extends previous works, preserving and improving the capability to generate overall plausible aggregated dynamics, but also significantly improving the precision in the rendering of microscopic group dynamics, with particular attention to the shape of dyads. The model has been calibrated and validated thanks to empirical data gathered in an experimental observation; both quantitative and qualitative achieved results are presented and discussed. Results show that the proposed approach, model and defined set of relevant parameters produce plausible results across a relatively large spectrum of situations, especially pedestrian density, although the discrete nature of the approach limits the possibility to achieve smooth and directly comparable trajectories and interpersonal distances among members of a dyad.

The model allows investigating situations for which we still lack empirical evidences, in particular high density pedestrian populations including groups. Results of simulations in these conditions call for additional real world experiments, to further evaluate the validity of this model. Additionally, we aim at using systematically the presented model to evaluate if empirically observed result such as the fact that obstacles can smoothen pedestrian flows in certain situations still hold in presence of groups. Extending the study to tactical level decisions, we will also investigate the effect of the presence of groups in 
wayfinding processes.

\section{Acknowledgements}

This research was supported by Key Research and Development Program (2016YFC0802508), Program of Shanghai Science and Technology Committee (16DZ1200106), Specialized Research Fund for the Doctoral Program of Higher Education of China (20133402110009) the China Scholarship Council (CSC) and Fundamental Research Funds for the Central Universities (WK2320000035).

\section{References}

[1] R. Challenger, C. W. Clegg, M. A. Robinson, Understanding Crowd Behaviours: Supporting Evidence, Tech. Rep., University of Leeds, 2009.

[2] A. Willis, N. Gjersoe, C. Havard, J. Kerridge, R. Kukla, Human movement behaviour in urban spaces: implications for the design and modelling of effective pedestrian environments, Environment and Planning B: Planning and Design 31 (6) (2004) 805-828, ISSN 0265-8135.

[3] M. Moussaïd, N. Perozo, S. Garnier, D. Helbing, G. Theraulaz, The walking behaviour of pedestrian social groups and its impact on crowd dynamics., PloS one 5 (4) (2010) e10047.

[4] G. Vizzari, L. Manenti, L. Crociani, Adaptive Pedestrian Behaviour for the Preservation of Group Cohesion, Complex Adaptive Systems Modeling $1(7)$.

[5] S. Bandini, L. Crociani, A. Gorrini, G. Vizzari, An agent-based model of pedestrian dynamics considering groups: A real world case study, in: 2014 IEEE 17th International Conference on Intelligent Transportation Systems (ITSC), IEEE, 572-577, 2014.

[6] A. Gorrini, L. Crociani, C. Feliciani, P. Zhao, K. Nishinari, S. Bandini, Social Groups and Pedestrian Crowds: Experiment on Dyads in a Counter 
Flow Scenario, in: Proceedings of the 8th International Conference on Pedestrian and Evacuation Dynamics - PED2016, Hefei, China, October 17-21, 2016, 179-184, 2016.

[7] F. Zanlungo, D. c. v. Brščić, T. Kanda, Spatial-size scaling of pedestrian groups under growing density conditions, Phys. Rev. E 91 (2015) 062810.

[8] F. Zanlungo, Z. Yücel, D. Brščić, T. Kanda, N. Hagita, Intrinsic group behaviour: Dependence of pedestrian dyad dynamics on principal social and personal features, PLOS ONE 12 (11) (2017) 1-26, doi:10.1371/journal. pone.0187253, URL https://doi.org/10.1371/journal.pone.0187253.

[9] M. Twarogowska, P. Goatin, R. Duvigneau, Macroscopic modeling and simulations of room evacuation, Applied Mathematical Modelling 38 (24) (2014) 5781-5795.

[10] G. Lämmel, A. Seyfried, B. Steffen, Large-scale and microscopic: a fast simulation approach for urban areas, in: Transportation Research Board 93rd Annual Meeting, 14-3890, 2014.

[11] D. Helbing, P. Molnár, Social force model for pedestrian dynamics, Phys. Rev. E 51 (5) (1995) 4282-4286.

[12] A. Schadschneider, A. Kirchner, K. Nishinari, CA Approach to Collective Phenomena in Pedestrian Dynamics., in: S. Bandini, B. Chopard, M. Tomassini (Eds.), Cellular Automata, 5th International Conference on Cellular Automata for Research and Industry, ACRI 2002, vol. 2493 of Lecture Notes in Computer Science, Springer, 239-248, 2002.

[13] S. Bandini, M. L. Federici, G. Vizzari, Situated Cellular Agents Approach to Crowd Modeling and Simulation, Cybernetics and Systems 38 (7) (2007) 729-753.

[14] C. M. Henein, T. White, Agent-Based Modelling of Forces in Crowds., in: P. Davidsson, B. Logan, K. Takadama (Eds.), Multi-Agent and MultiAgent-Based Simulation, Joint Workshop MABS 2004, New York, NY, 
USA, July 19, 2004, Revised Selected Papers, vol. 3415 of Lecture Notes in Computer Science, Springer-Verlag, 173-184, 2005.

[15] S. R. Musse, D. Thalmann, Hierarchical Model for Real Time Simulation of Virtual Human Crowds, IEEE Trans. Vis. Comput. Graph. 7 (2) (2001) $152-164$.

[16] W. Shao, D. Terzopoulos, Autonomous pedestrians, Graphical Models 69 (5-6) (2007) 246-274.

[17] S. Paris, S. Donikian, Activity-Driven Populace: A Cognitive Approach to Crowd Simulation, IEEE Computer Graphics and Applications 29 (4) (2009) 34-43.

[18] E. T. Hall, The Hidden Dimension, Anchor Books, 1966.

[19] J. Was, Crowd Dynamics Modeling in the Light of Proxemic Theories, in: L. Rutkowski, R. Scherer, R. Tadeusiewicz, L. A. Zadeh, J. M. Zurada (Eds.), ICAISC (2), vol. 6114 of Lecture Notes in Computer Science, Springer, ISBN 978-3-642-13231-5, 683-688, 2010.

[20] T. Ezaki, D. Yanagisawa, K. Ohtsuka, K. Nishinari, Simulation of space acquisition process of pedestrians using Proxemic Floor Field Model, Physica A 391 (1-2) (2012) 291 - 299, ISSN 0378-4371.

[21] L. Manenti, S. Manzoni, G. Vizzari, K. Ohtsuka, K. Shimura, An AgentBased Proxemic Model for Pedestrian and Group Dynamics: Motivations and First Experiments, in: D. Villatoro, J. Sabater-Mir, J. S. Sichman (Eds.), MABS, vol. 7124 of Lecture Notes in Computer Science, Springer, ISBN 978-3-642-28399-4, 74-89, 2011.

[22] M. Moussaïd, N. Perozo, S. Garnier, D. Helbing, G. Theraulaz, The Walking Behaviour of Pedestrian Social Groups and Its Impact on Crowd Dynamics, PLoS ONE 5 (4) (2010) e10047. 
[23] S. Xu, H. B.-L. Duh, A Simulation of Bonding Effects and Their Impacts on Pedestrian Dynamics, IEEE Transactions on Intelligent Transportation Systems 11 (1) (2010) 153-161.

[24] G. Köster, M. Seitz, F. Treml, D. Hartmann, W. Klein, On modelling the influence of group formations in a crowd, Contemporary Social Science 6 (3) (2011) 397-414.

[25] F. Müller, O. Wohak, A. Schadschneider, Study of Influence of Groups on Evacuation Dynamics Using a Cellular Automaton Model, Transportation Research Procedia 2 (2014) 168 - 176, ISSN 2352-1465, the Conference on Pedestrian and Evacuation Dynamics 2014 (PED 2014), 22-24 October 2014, Delft, The Netherlands.

[26] F. Qiu, X. Hu, Modeling group structures in pedestrian crowd simulation, Simulation Modelling Practice and Theory 18 (2) (2010) 190-205, ISSN 1569190X.

[27] R. A. Rodrigues, A. de Lima Bicho, M. Paravisi, C. R. Jung, L. P. Magalhães, S. R. Musse, An Interactive Model for Steering Behaviors of Groups of Characters, Applied Artificial Intelligence 24 (6) (2010) 594-616.

[28] G. Vizzari, L. Manenti, K. Ohtsuka, K. Shimura, An Agent-Based Pedestrian and Group Dynamics Model Applied to Experimental and Real-World Scenarios, J. Intellig. Transport. Systems 19 (1) (2015) 32-45.

[29] C. von Krüchten, A. Schadschneider, Empirical study on social groups in pedestrian evacuation dynamics, Physica A 475 (2017) 129 - 141.

[30] N. W. F. Bode, S. Holl, W. Mehner, A. Seyfried, Disentangling the Impact of Social Groups on Response Times and Movement Dynamics in Evacuations, PLOS ONE 10 (3) (2015) 1-14.

[31] A. Templeton, J. Drury, A. Philippides, From mindless masses to small groups: Conceptualizing collective behavior in crowd modeling., Review of General Psychology 19 (3) (2015) 215. 
[32] S. Bandini, L. Crociani, G. Vizzari, An Approach for Managing Heterogeneous Speed Profiles in Cellular Automata Pedestrian Models, Journal of Cellular Automata 12 (5) (2017) 401-421.

[33] D. Weyns, A. Omicini, J. Odell, Environment as a First Class Abstraction in Multiagent Systems, Autonomous Agents Multi-Agent Systems 14 (1) (2007) $5-30$.

[34] U. Weidmann, Transporttechnik der Fussgänger - Transporttechnische Eigenschaftendes Fussgängerverkehrs (Literaturstudie), Literature Research 90, Institut füer Verkehrsplanung, Transporttechnik, Strassen- und Eisenbahnbau IVT an der ETH Zürich, 1993.

[35] S. Bandini, M. Mondini, G. Vizzari, Modelling negative interactions among pedestrians in high density situations, Transportation Research Part C: Emerging Technologies 40 (0) (2014) 251 - 270, ISSN 0968-090X.

[36] C. Burstedde, K. Klauck, A. Schadschneider, J. Zittartz, Simulation of pedestrian dynamics using a two-dimensional cellular automaton, Physica A: Statistical Mechanics and its Applications 295 (3 - 4) (2001) 507-525, ISSN 0378-4371.

[37] E. T. Hall, The hidden dimension, Doubleday New York Ed., 1966.

[38] S. Bandini, L. Crociani, G. Vizzari, Heterogeneous Pedestrian Walking Speed in Discrete Simulation Models, in: M. Chraibi, M. Boltes, A. Schadschneider, A. Seyfried (Eds.), Traffic and Granular Flow '13, Springer International Publishing, ISBN 978-3-319-10628-1, 273-279, 2015.

[39] A. Kirchner, H. Klüpfel, K. Nishinari, A. Schadschneider, M. Schreckenberg, Discretization effects and the influence of walking speed in cellular automata models for pedestrian dynamics, Journal of Statistical Mechanics: Theory and Experiment 2004 (10) (2004) P10011.

[40] S. Koyama, N. Shinozaki, S. Morishita, Pedestrian flow modeling using cellular automata based on the Japanese public guideline and application 
to evacuation simulation, Journal of Cellular Automata 8 (5-6) (2013) 361382.

[41] L. Crociani, G. Lämmel, Multidestination Pedestrian Flows in Equilibrium: A Cellular Automaton-Based Approach, Computer-Aided Civil and Infrastructure Engineering 31 (6) (2016) 432-448.

[42] A. Kirchner, K. Nishinari, A. Schadschneider, Friction effects and clogging in a cellular automaton model for pedestrian dynamics, Phys. Rev. E 67 (5) (2003) 56122 .

[43] A. Kirchner, A. Namazi, K. Nishinari, A. Schadschneider, Role of Conflicts in the Floor Field Cellular Automaton Model for Pedestrian Dynamics, in: 2nd International Conference on Pedestrian and Evacuation Dynamics, $51-62,2003$.

[44] T. Ezaki, D. Yanagisawa, K. Nishinari, Pedestrian flow through multiple bottlenecks, Physical Review E - Statistical, Nonlinear, and Soft Matter Physics 86 (2) (2012) 026118, ISSN 15393755.

[45] Y. Suma, D. Yanagisawa, K. Nishinari, Anticipation effect in pedestrian dynamics: Modeling and experiments, Physica A: Statistical Mechanics and its Applications 391 (1-2) (2012) 248-263, ISSN 03784371.

[46] C. J. E. Castle, N. P. Waterson, E. Pellissier, S. Bail, A Comparison of Gridbased and Continuous Space Pedestrian Modelling Software: Analysis of Two UK Train Stations, in: R. D. Peacock, E. D. Kuligowski, J. D. Averill (Eds.), Pedestrian and Evacuation Dynamics, Springer US, ISBN 978-14419-9724-1, 433-446, 2011.

[47] M. Boltes, A. Seyfried, Collecting pedestrian trajectories, Neurocomputing 100 (2013) 127-133, ISSN 09252312. 